\title{
APS-TD Quality Facilities and Tools
}

\section{Jamie Blowers, APS-TD Quality \& Materials Department}

FERMILAB-POSTER-20-032-TD

\section{Quality Function Organization}

In the Applied Physics and Superconducting Technology Division the quality function resides within the Quality \& Materials Department (QMD). QMD employs staff with expertise in qualityrelated fields and activities such as:

- Quality Programs (e.g. DOE, ISO) both for operations and for projects

- Metrology

- Application of the Geometric Dimensioning \& Tolerancing (GD\&T) Code, both for designs and for inspections

- Failure Modes \& Effects Analysis (FMEA)

- Process Engineering

- High-vacuum leak checking

- Electrical (R, L, Q) testing

- Hydrostatic and flow testing

- Calibration

\section{World-Class Process Quality Tool}

In addition to expertise in measurements, we also developed an electronic traveler system, which is used for all travelers in APSTD, along with Mu2e, LCLS-II, HiLumi LHC Upgrade Project (including work at BNL and LBNL), PIP-II, and LCLS-II HE.

The tool, Vector, has world read-only access for the purposes of sharing data amongst multi-site collaborations:

https://vector.fnal.gov/

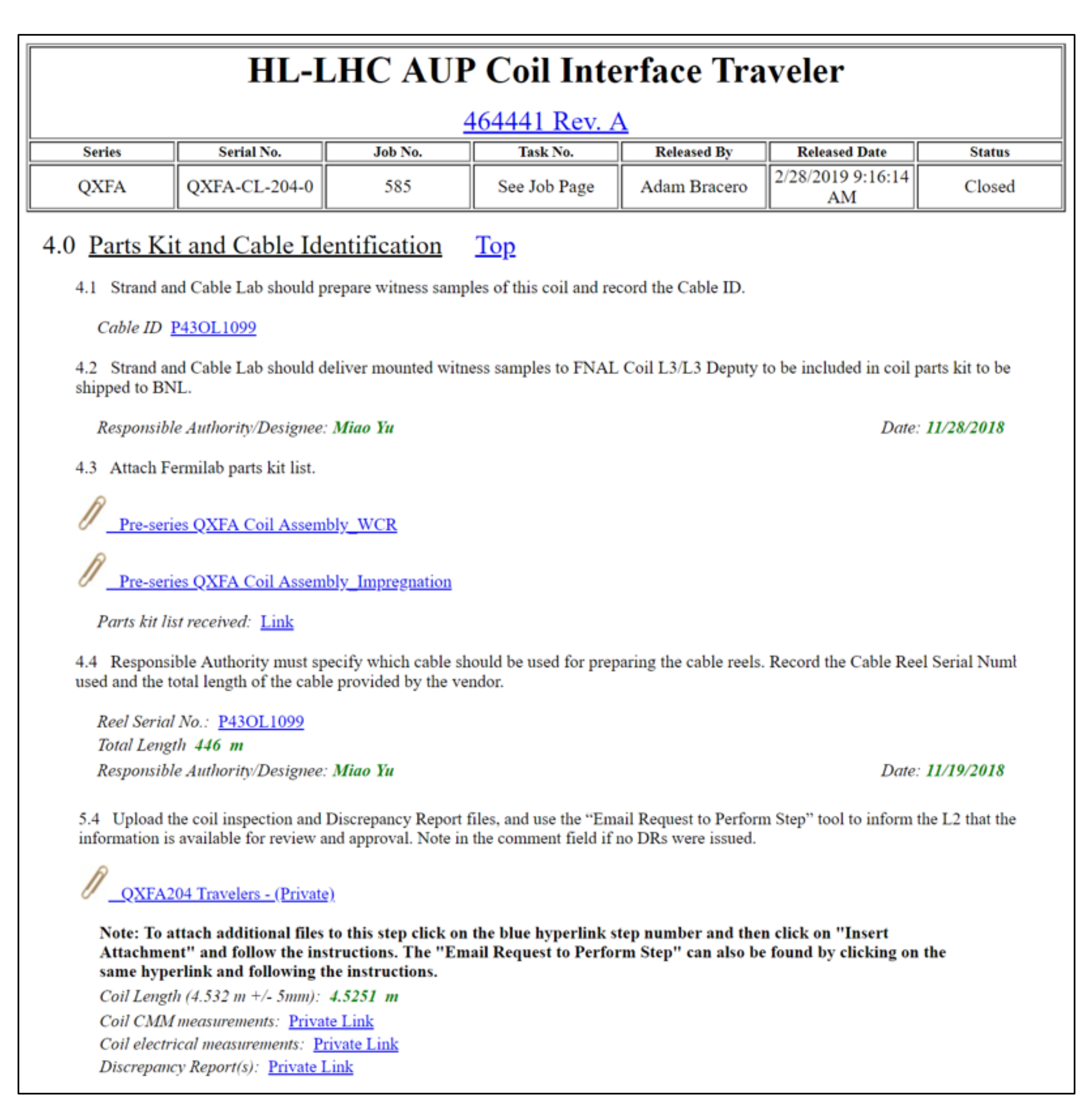

Example electronic traveler

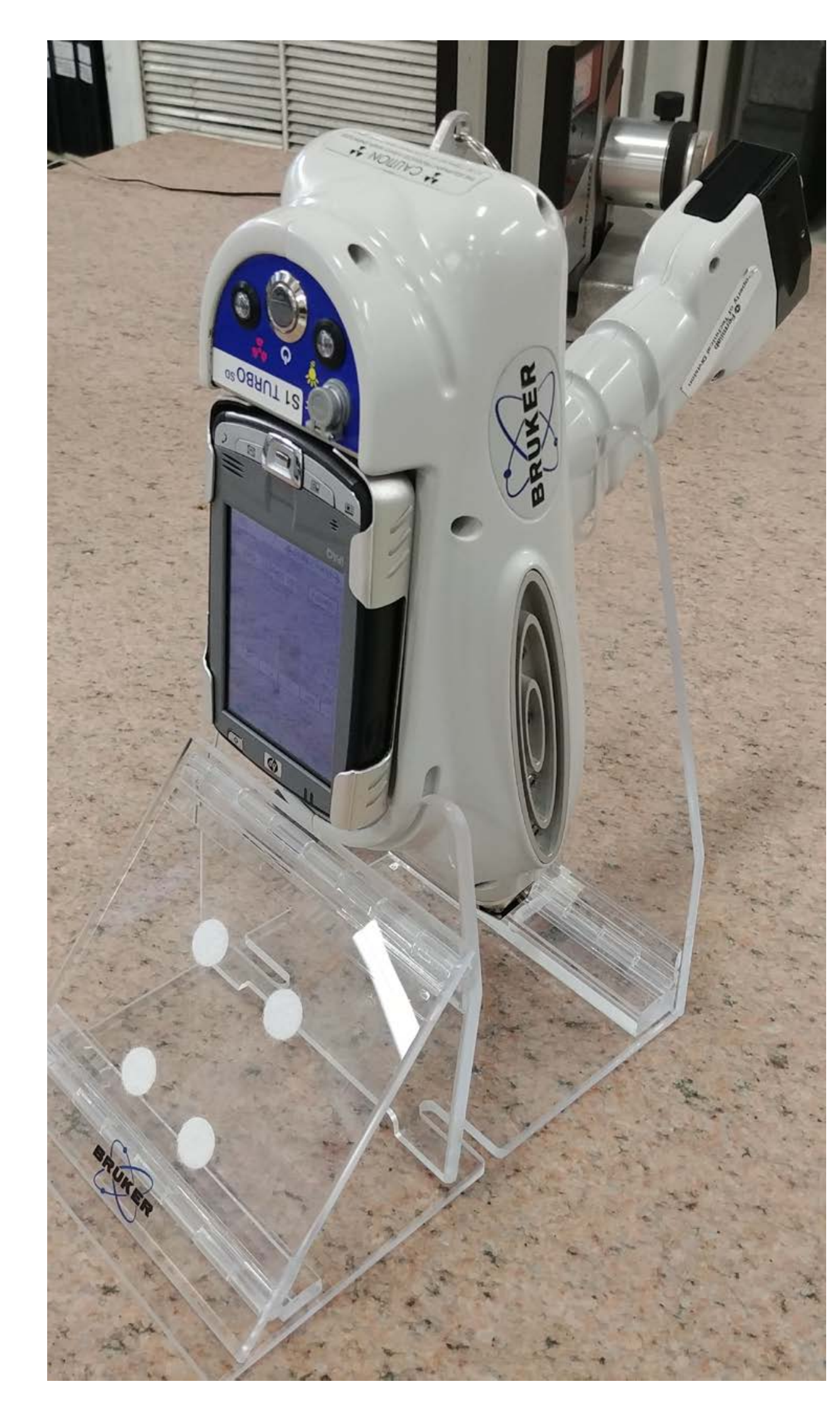

Handheld Alloy Analyzer

\section{Inspection Equipment}

The department maintains and uses a wide range of inspection and measurement equipment in the support of the division, lab, and the projects. This equipment includes:

- Coordinate Measurement Machines, including the use of Scanning Probes to generate point clouds

- Laser Tracker

- Optical Comparators

- Alloy Analyzer

- Magnetic Permeability

- Leak checking and vacuum equipment, both conventional and contra-flow, and dry or oil-based

- Hipoters, Ringers, RLQ measurement tools

- Hydrostatic and flow carts

- A plethora of hand gauges

\section{Services Offered}

While our primary customers are within APS-TD, our services are offered to the entire laboratory as the primary resource within the lab with the breadth of skills, expertise, and facilities for highprecision measurements and process controls.

These tools and skills are suitable for work in the support of accelerator operations, R\&D, as well as DOE O 413 Projects.

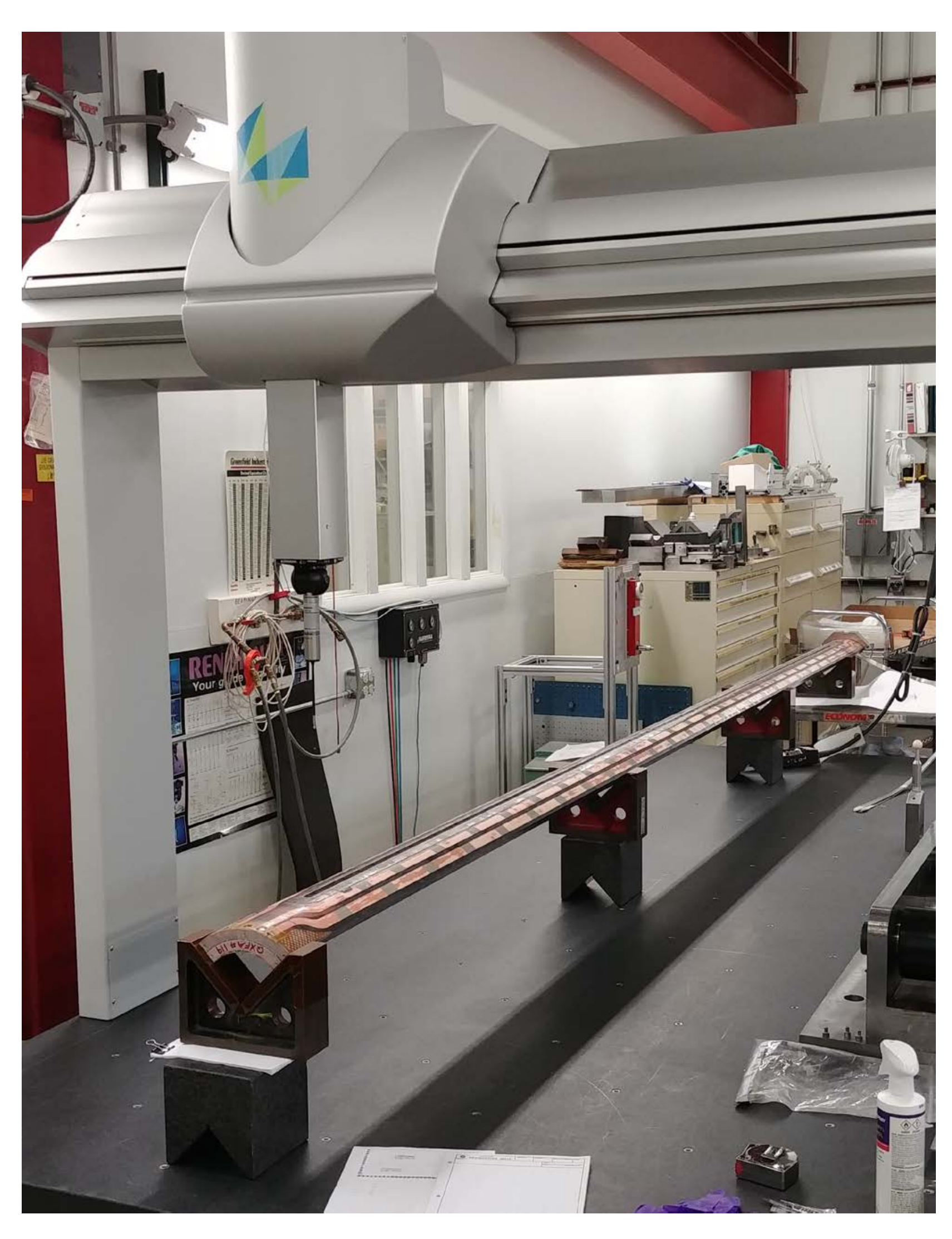

CMM measuring HiLumi LHC coil

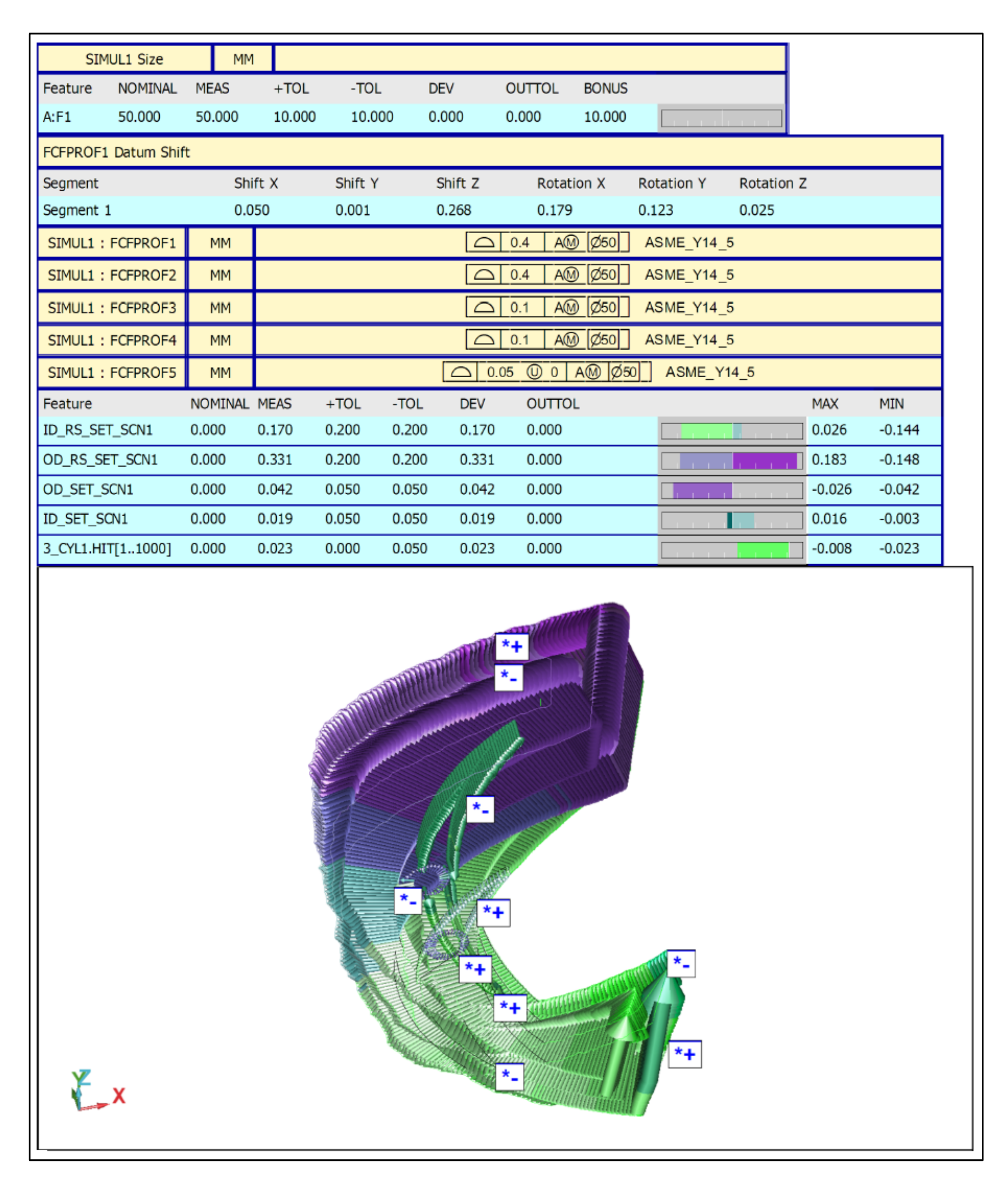

Example CMM inspection report 